\title{
The Role of Northern Sea Ice Cover for the Weakening of the Thermohaline Circulation under Global Warming
}

\author{
A. Levermann, J. Mignot, * S. Nawrath, and S. Rahmstorf \\ Potsdam Institute for Climate Impact Research, Potsdam, Germany
}

(Manuscript received 17 July 2006, in final form 20 December 2006)

\begin{abstract}
An increase in atmospheric $\mathrm{CO}_{2}$ concentration and the resulting global warming are typically associated with a weakening of the thermohaline circulation (THC) in model scenarios. For the models participating in the Coupled Model Intercomparison Project (CMIP), this weakening shows a significant $(r=0.62)$ dependence on the initial THC strength; it is stronger for initially strong overturning. The authors propose a physical mechanism for this phenomenon based on an analysis of additional simulations with the coupled climate models CLIMBER-2 and CLIMBER-3 $\alpha$. The mechanism is based on the fact that sea ice cover greatly reduces heat loss from the ocean. The extent of sea ice is strongly influenced by the near-surface atmospheric temperature (SAT) in the North Atlantic but also by the strength of the THC itself, which transports heat to the convection sites. Consequently, sea ice tends to extend farther south for weaker THC. Initially larger sea ice cover responds more strongly to atmospheric warming; thus, sea ice retreats more strongly for an initially weaker THC. This sea ice retreat tends to strengthen (i.e., stabilize) the THC because the sea ice retreat allows more oceanic heat loss. This stabilizing effect is stronger for runs with weak initial THC and extensive sea ice cover. Therefore, an initially weak THC weakens less under global warming. In contrast to preindustrial climate, sea ice melting presently plays the role of an external forcing with respect to THC stability.
\end{abstract}

\section{Introduction}

The thermohaline circulation (THC) transports about $1 \mathrm{PW}\left(=10^{15} \mathrm{~W}\right)$ of heat to the North Atlantic (Hall and Bryden 1982; Ganachaud and Wunsch 2000, 2003), having a major effect on atmospheric and oceanic temperatures in northern high latitudes (e.g., Manabe and Stouffer 1988; Wood et al. 1999). In addition to these regional effects, the overturning circulation influences climate on a global scale (Winton 2003; Herweijer et al. 2005). Because of the associated heat transport, the thermal equator is shifted northward over the Atlantic and eastern Pacific. The intertropical convergence zone follows this excursion, which leads to altered precipitation patterns compared to a climate state without overturning (Vellinga and Wood 2002; Stouffer et al. 2006). The existence of an interhemi-

\footnotetext{
* Current affiliation: LOCEAN, Paris, France.
}

Corresponding author address: A. Levermann, Potsdam Institute for Climate Impact Research, Telegrafenberg A26, 14473 Potsdam, Germany.

E-mail: anders.levermann@pik-potsdam.de spheric overturning circulation yields both a sea surface elevation that is some decimeters lower in the North Atlantic compared to the North Pacific (Levermann et al. 2005) and a cooling of the Southern Hemisphere (Crowley 1992; Stocker 1998). It has strong implications for the marine ecosphere (e.g., Schmittner 2005) and possible teleconnection with the El Niño-Southern Oscillation (ENSO) phenomenon (Timmermann et al. 2005). Combined evidence from proxy data (e.g., McManus et al. 2004) and simulations (e.g., Manabe and Stouffer 1997) suggests that large and abrupt changes in THC have led to major climate shifts during the last glacial period [for a detailed discussion see the reviews by Clark et al. (2002) and Rahmstorf (2002)]. Past cessations of the Atlantic overturning have been associated with massive freshwater release from glaciers to the ocean (Bond et al. 1992).

In light of future climate change, an understanding of the stability properties of the THC is of particular importance. Projections of future climate indicate a crucial role of Greenland meltwater flux for the evolution of the THC (Fichefet et al. 2003). However, some models show a pronounced reduction of overturning that is not due to meltwater inflow. For example, the atmo-

DOI: $10.1175 /$ JCLI4232.1

(C) 2007 American Meteorological Society 
sphere-ocean general circulation model (AOGCM), ECHAM3/LSG, exhibits a complete collapse of deepwater formation for $1 \%$ of yearly $\mathrm{CO}_{2}$ increase and a subsequent stabilization at $1120 \mathrm{ppm}$ (Winguth et al. 2005 ). Though the model was coupled to a land ice model and therefore included Greenland meltwater flux, the slowdown of overturning was induced mainly by an increase in precipitation in the North Atlantic of $\sim 0.2 \mathrm{~Sv}\left(1 \mathrm{~Sv}=10^{6} \mathrm{~m}^{3} \mathrm{~s}^{-1}\right)$ over a period of several hundred years. On shorter time scales, most coupled climate models without Greenland meltwater inflow do not show a complete THC collapse. In recent intercomparison projects (Gregory et al. 2005; Petoukhov et al. 2005), this weakening was gradual in all participating models and varied between $10 \%$ and $50 \%$ of the initial value.

On decadal or shorter time scales, changes in THC will be strongly influenced by changes in North Atlantic buoyancy flux because of their impact on deep-water formation. Saenko et al. (2004) examine the role of northern sea ice cover for the overturning circulation during global warming experiments by altering the thermal diffusion coefficient in their atmospheric energy-moisture balance model, thereby producing varying temperature and sea ice extent in northern high latitudes. Their main conclusion is that the initial climate around the subpolar gyre is crucial for understanding the weakening of the THC under global warming. In their experiments with moderately cold initial temperatures in the north, they observe a weakening of the THC, while it strengthens when northern high latitudes are unrealistically cold and sea ice extends farther south.

Because of a lack of observational data, limitations of currently available climate models, and natural variability, the present strength of the Atlantic meridional overturning circulation is poorly constrained (Ganachaud and Wunsch 2000, 2003; Talley et al. 2003; Zickfeld et al. 2007). The maximum overturning of the participating models of the Coupled Model Intercomparison Project (CMIP; http://www.gfdl.noaa.gov/ kd/ CMIP.html) varies between $\sim 12$ and $\sim 27 \mathrm{~Sv}$ and the Third Assessment Report of the Intergovernmental Panel for Climate Change (IPCC TAR; Houghton et al. 2001) gives a range of even $\sim 10$ to $\sim 30 \mathrm{~Sv}$ between the participating models. In view of this uncertainty, the dependence of the sensitivity of the THC to an increase in atmospheric $\mathrm{CO}_{2}$ on its initial strength is an important issue. Using the Earth System Models of Intermediate Complexity (EMICs) CLIMBER-2 and CLIMBER-3 $\alpha$ and results from the CMIP simulations (section 2), we investigate this dependence (section 3) and propose a physical mechanism based on the stabi- lizing effect of changes in sea ice cover on the THC (section 4). The results are consistent with simulations carried out with a number of AOGCMs in the framework of the IPCC Fourth Assessment Report (IPCC AR4). Section 5 qualitatively discusses the phenomenon in physical terms. Conclusions and a discussion, particularly with respect to a generalization of the mechanism to other models, are given in section 6 .

\section{Models and experiments}

To understand the influence of the initial strength of the overturning on its change under global warming, 14 simulations using the EMICs CLIMBER-2 and CLIMBER-3 $\alpha$ were carried out, as a complement to the CMIP runs. CLIMBER-2 is a fully coupled climate model composed of a statistical-dynamical atmosphere module and zonally averaged ocean and sea ice models. The sea ice module is based on the one-layer thermodynamic (Semtner 1976) model with simple treatment of advection and diffusion. It calculates the sea ice fraction and thickness based on the sea ice mass balance and a relation between ice thickness and fraction. The ice is freely transported (no ice rheology) by advection and horizontal diffusion. CLIMBER-2 has been used to simulate present day climate (Petoukhov et al. 2000) and the last glacial maximum (Kubatzki et al. 2000), as well as abrupt climatic events of the past (Ganopolski and Rahmstorf 2001) and future scenarios (Rahmstorf and Ganopolski 1999). With its coarse resolution and therefore fast performance, the model is suited for very long integrations including equilibria and large ensemble simulations (e.g., von Deimling et al. 2006).

The EMIC CLIMBER-3 $\alpha$ uses the same atmospheric module as CLIMBER-2 with a higher horizontal resolution and explicit topography. The ocean component is based on the Geophysical Fluid Dynamics Laboratory (GFDL) OGCM Modular Ocean Model (MOM-3) with a horizontal resolution of $3.75^{\circ} \times 3.75^{\circ}$ and 24 variably spaced vertical levels. The sea ice model was adopted from Fichefet and Maqueda (1997). It is a twodimensional (latitude-longitude) thermodynamic-dynamic model with one layer of ice and one layer of snow. It takes into account the heat capacity of snow and ice, the thermodynamic effects of the subgrid-scale snow and ice thickness distributions, the storage of latent heat in brine pockets, and the formation of snow ice. The sea ice dynamics allows the sea ice to flow under the effect of winds and currents and to selfinteract. The dynamics employs an elasto-visco-plastic rheology formulation, thereby conserving mechanical energy. For a full description of CLIMBER-3 $\alpha$, see Montoya et al. 2005; the sensitivity of the model to 
TABLE 1. Simulations carried out in the framework of the IPCC AR4.

\begin{tabular}{ll}
\hline \hline Acronym & \multicolumn{1}{c}{ Model description } \\
\hline CCCMA & $\begin{array}{c}\text { Canadian Centre for Climate Modelling and } \\
\text { Analysis; CGCM3.1 Model, T47 resolution } \\
\text { ECHO }\end{array}$ \\
$\begin{array}{c}\text { Meteorological Institute of the University of Bonn; } \\
\text { ECHO-G Model }\end{array}$ \\
GFDL & NOAA Geophysical Fluid Dynamics Laboratory; \\
& CM2.1 Model \\
GISS & NASA Goddard Institute for Space Studies; \\
& ModelE20/Russell \\
IPSL & IPSL/LMD/LSCE, France; CM4 V1 Model \\
MIROC & CCSR/NIES/FRCGC, Japan; MIROC3.2, medium \\
& resolution, 3 simulations \\
\hline
\end{tabular}

vertical diffusivity was investigated by Mignot et al. (2006).

Both of these EMICs took part in the CMIP study together with six AOGCMs and three other EMICs. A complete list of the participating models is given in Table 1 of Stouffer et al. 2006. The CMIP contained a number of idealized experiments designed to investigate the models' response to a quadrupling of atmospheric $\mathrm{CO}_{2}$ (Gregory et al. 2005) and an anomalous freshwater flux to the northern Atlantic (Stouffer et al. 2006). In this study we concentrate on the scenario runs with a $1 \%$ increase per year of atmospheric $\mathrm{CO}_{2}$ concentration over $140 \mathrm{yr}$ up to $1120 \mathrm{ppm}$ (i.e., 4 times the preindustrial value of $280 \mathrm{ppm}$; denoted "standard experiment" hereafter). It should be noted that none of these experiments included meltwater from continental ice, which could become a major source of freshwater if the Greenland Ice Sheet melts rapidly (Alley et al. 2005; Oppenheimer and Alley 2005; Hansen 2005).

The initial strength of the overturning circulation in the participating CMIP models varies between $\sim 12$ and $\sim 27 \mathrm{~Sv}$. To investigate the importance of this initial scatter, we performed additional experiments. The initial strength of the THC was varied by application of a constant anomalous freshwater flux to the Atlantic Ocean surface both in CLIMBER-2 and CLIMBER$3 \alpha$. The flux was applied in the North Atlantic between $50^{\circ}$ and $70^{\circ} \mathrm{N}$ and compensated in the Pacific between $45^{\circ} \mathrm{S}$ and $55^{\circ} \mathrm{N}$. With anomalous freshwater fluxes between -0.15 and $+0.09 \mathrm{~Sv}$, we obtained 11 initial equilibria with a range of 16.6-26.0 Sv of maximum overturning in CLIMBER-2. The standard value is $21.6 \mathrm{~Sv}$. Anomalous freshwater fluxes of -0.1 and $-0.2 \mathrm{~Sv}$ in CLIMBER-3 $\alpha$ resulted in THC maxima of 13.2 and $16.2 \mathrm{~Sv}$, respectively, compared to the standard value of $11.8 \mathrm{~Sv}$ without anomalous freshwater forcing. This way, we cover the entire interval of the CMIP simulations.
To test the robustness of the physical mechanism proposed in this study (section 4), we use simulations that were carried out in the framework of the IPCC AR4. In particular, we analyze simulations of AOGCMs, for which overturning strength and sea ice concentration for the standard experiment is provided (wwwpcmdi.llnl.gov/ipcc/about_ipcc.php; sea ice data are not available for the CMIP.). Table 1 gives the simulations used here.

\section{THC sensitivity to atmospheric $\mathrm{CO}_{2}$ increase: Dependence on initial overturning strength}

Figure 1 shows the time evolution of the overturning strength in CLIMBER-2 (black) and CLIMBER-3 $\alpha$ (colors) runs with different initial THC strength for the standard experiment. The $\mathrm{CO}_{2}$ concentration is shown as the dashed curve. As a consequence of increased $\mathrm{CO}_{2}$ forcing, all simulations show a rapid reduction of the THC. When the atmospheric $\mathrm{CO}_{2}$ concentration is kept constant, the THC gradually recovers. After the final $\mathrm{CO}_{2}$ concentration is reached (140 yr after the onset of the scenario), the interval of initial volume transport $M_{0} \in[11.8,26.0]$ Sv reduces to values $M_{140} \in$ $[9.6,17.4] \mathrm{Sv}$ and becomes $M_{2000} \in[13.6,20.4] \mathrm{Sv}$ in the final equilibrium (2000 yr after the start of the scenario). The shrinking of the interval with time from 14.2 via $7.8 \mathrm{~Sv}$ to $6.8 \mathrm{~Sv}$ reflects the fact that the $\mathrm{CO}_{2}$ sensitivity of the THC depends on its initial strength $M_{0}$. Figure 2 gives the THC weakening $\Delta M_{t}=M_{0}-M_{t}$ as a function of $M_{0}$, after $t=70 \mathrm{yr}$ (top panel), $t=140 \mathrm{yr}$ (middle panel), and for the final equilibrium $\Delta M_{2000}$ (bottom panel). For each of these time slices, the results of the simulations with CLIMBER-2 (black dots) and CLIMBER-3 $\alpha$ (colored dots) exhibit a quasi-linear relationship of $\Delta M_{t}$ with the initial THC strength $M_{0}$ (correlation coefficients of the linear regression $r \in[0.97$, 0.99]). The other CMIP simulations (red crosses) and the IPCC AR4 simulations (blue crosses) scatter around these lines. Linear regression yields a correlation coefficient $r=0.63$ for a fit of the CMIP runs alone and $r=0.60$ for the CMIP and IPCC AR4 experiments. The correlation coefficient increases to $r=0.72$ when all available simulations in the middle panel of Fig. 2 are taken into account. Simulations that were carried out in the framework of the EMIC Intercomparison Project (EMIP; Petoukhov et al. 2005) exhibit a similar scatter around the CLIMBER simulations (top panel, green crosses). Here the $1 \% \mathrm{CO}_{2}$ increase scenario was integrated only up to a doubling of the preindustrial concentration, $560 \mathrm{ppm}$.

Note that in the course of the scenario, the overturning eventually becomes stronger in the two CLIMBER- 


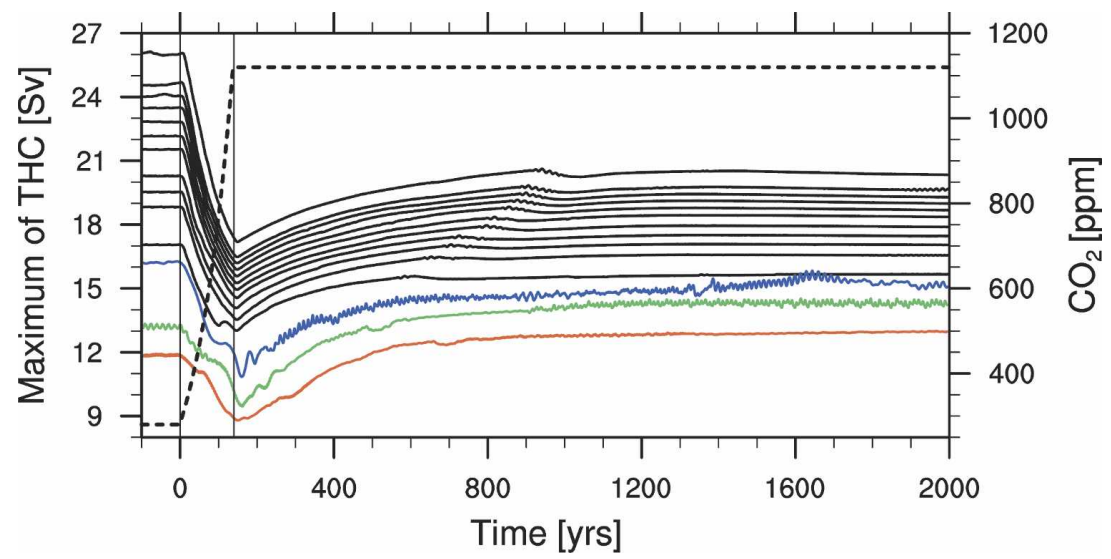

FIG. 1. Maximum of the THC from $100 \mathrm{yr}$ before to $2000 \mathrm{yr}$ after the start of the $\mathrm{CO}_{2}$ increase. Eleven simulations with CLIMBER-2 are shown in black and the three runs with CLIMBER-3 $\alpha$ are in color. Note that the weakening is stronger for stronger initial value. For the two weakest initial THC, even a strengthening in the final equilibrium is observed. The atmospheric $\mathrm{CO}_{2}$ concentration rises within $140 \mathrm{yr}$ from 280 to $1120 \mathrm{ppm}$ and stays constant thereafter (dashed curve, right axis).

$3 \alpha$ simulations with weakest initial THC. Consequently, the line formed by the CLIM $B E R$ equilibrium simulations (Fig. 2, bottom panel) crosses the $x$ axes $\Delta M_{t}=0$ $\mathrm{Sv}$ for finite initial overturning strength $M_{2000}^{(0)}=$ $\sim 15 \mathrm{~Sv}$. For the two earlier time slices, the transition toward a strengthening occurs for $M_{140}^{(0)}=\sim 11 \mathrm{~Sv}$ and $M_{70}^{(0)}=\sim 8 \mathrm{~Sv}$ (Fig. 2, middle and top panel). Note also that the linear behavior of $\Delta M_{t}$ with $M_{0}$ occurs throughout the entire scenario and is not a specific feature of the selected time slices. For warming surface air temperature (SAT), that is, in the course of the $\mathrm{CO}_{2}$ scenario, the slope of the curves increases (cf. top and middle panel of Fig. 2). After $140 \mathrm{yr}$ (i.e., when $\mathrm{CO}_{2}$ is fixed to $1120 \mathrm{ppm}$ ), the slope slightly declines and stabilizes at 0.51 (full evolution with time not shown).

\section{The role of initial THC strength and northern sea ice cover}

In this section we propose a physical mechanism that leads to the qualitative behavior seen in Fig. 2. In the CMIP, the standard experiment was complemented by runs, where freshwater fluxes to the ocean in the control and scenario simulation were switched (Gregory et al. 2005; cf. Fig. 3). This experimental setup provides a linear approach to determine the comparative influence of changes in freshwater and heat forcing during the scenario. Gregory et al. (2005) showed that in the CMIP simulations, the changes in heat flux dominate the weakening of the THC in all models (see their Fig. $4)$. The freshwater fluxes include evaporation, precipitation, and river runoff as well as fluxes from produc- tion and the melting of sea ice. The dominance of heat flux changes is particularly pronounced in CLIMBER-2 (Fig. 3), while in other models freshwater fluxes may have a stronger influence. Focusing here on the ensemble of CLIMBER-2 simulations, we will therefore propose a purely heat flux-related mechanism, while keeping in mind that the influence of freshwater flux plays a secondary, but possibly nonnegligible role in other models. Limitations of a generalization of the mechanism to other models and the real climate system will be discussed in section 6 .

\section{a. Primary effects of $\mathrm{CO}_{2}$ increase on THC strength}

In boreal winter, the atmosphere in high northern latitudes is much colder than the freezing point. Thus, the area of extreme oceanic heat loss follows closely the ice-free area $A$, while heat loss is relatively weak underneath sea ice. This is illustrated in Fig. 4, where heat flux from atmosphere to ocean and sea ice fraction are plotted as functions of latitude for different initial THC strength in CLIMBER-2. Shown are zonal and annual mean at the beginning of the scenario $(t=0)$ for the North Atlantic. The sea ice edge and the area of intense oceanic heat loss retreat northward with increasing $M_{0}$ due to stronger northward heat transport by the overturning. In contrast, the southern border of negative heat flux $\left(\sim 20^{\circ} \mathrm{N}\right)$ is independent of the initial THC strength $M_{0}$.

The atmosphere-ocean heat flux is composed of latent, radiative, and turbulent heat fluxes, which all depend on the ice-free area $A$ and temperature difference between ocean and atmosphere $\Delta T \equiv \mathrm{T}_{o}-T_{a}$. To first 

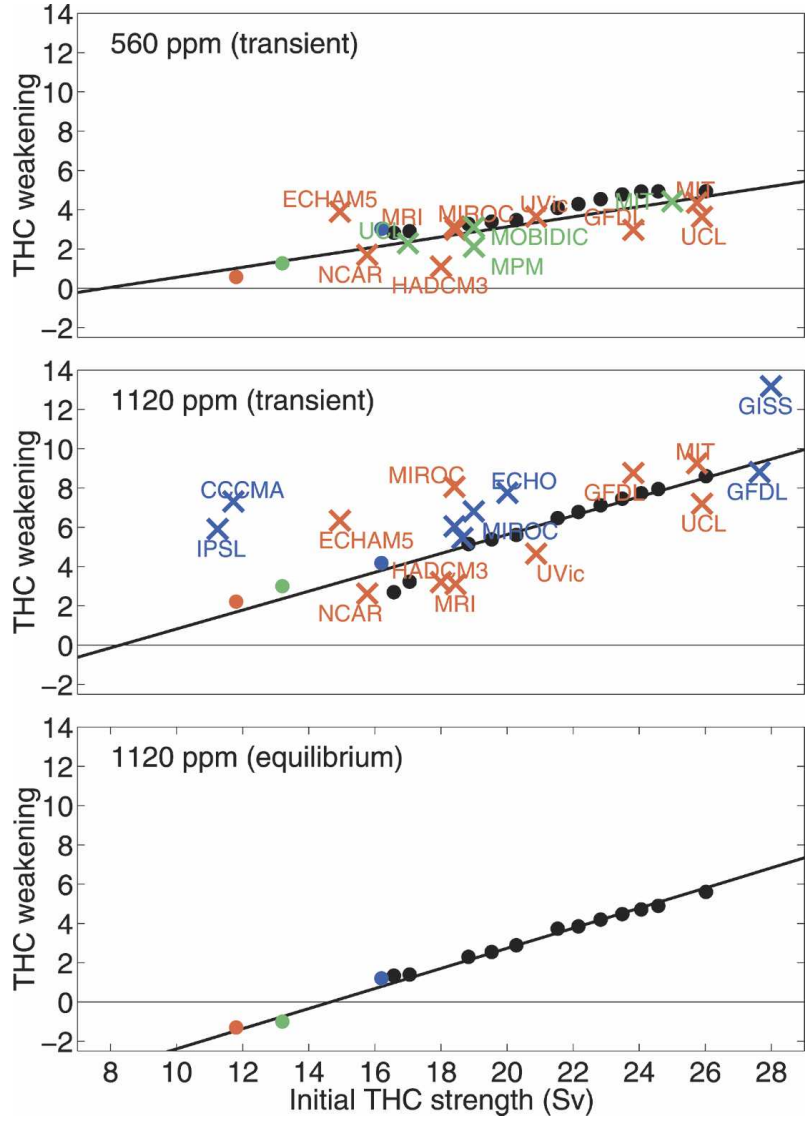

FIG. 2. Change in THC strength after (top) $70 \mathrm{yr}$, (middle) 140 $\mathrm{yr}$, and (bottom) in $1120 \mathrm{ppm} \mathrm{CO}_{2}$ equilibrium relative to the initial THC (CLIMBER-2: black dots and CLIMBER-3 $\alpha$ : colored dots) compared to participating CMIP (red crosses), EMIP (green crosses), and IPCC AR4 (blue crosses) models. The CMIP and EMIP models scatter around the lines given by the CLIMBER simulations through linear regression. The intersection with the $x$ axis gives the initial THC strength $M_{t}^{(0)}$ that does not exhibit change for the specific $\mathrm{CO}_{2}$ concentration.

order, the heat loss of the ocean $H$ is thus proportional to the product of the two quantities:

$$
H \approx \alpha_{H} \times A \times\left(T_{o}-T_{a}\right)=\alpha_{H} \times A \times \Delta T,
$$

where $\alpha_{H}$ is a positive proportionality constant. Note that other factors such as shortwave radiation may influence $H$. We restrict our discussion to situations where Eq. (1) holds. A reduction in oceanic heat loss $H$ decreases the oceanic surface density in the northern convection sites and leads to a weakening of North Atlantic Deep Water (NADW) formation and THC strength. We will now successively examine the effect of changes in temperature and sea ice cover on $H$ in the course of the standard experiment.

Let us first consider the effect of temperature changes. Figure 5 shows the time series of the differ-

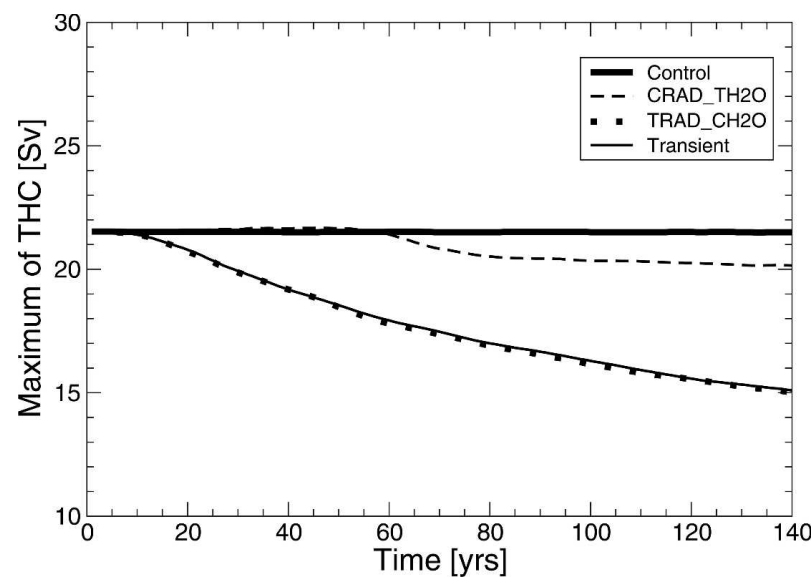

FIG. 3. THC during the 4 CMIP experiments with CLIMBER-2: the 140 -yr control simulation (black), the transient with $1 \%$ yearly $\mathrm{CO}_{2}$ increase, and the 2 experiments with exchanged freshwater flux to the ocean. In CRAD_TH2O, $\mathrm{CO}_{2}$ is constant and the freshwater flux from the transient run is applied, and vice versa in TRAD_CH2O.

ence between oceanic and atmospheric temperature in January averaged over the entire North Atlantic between $20^{\circ}$ and $80^{\circ} \mathrm{N}$. The lines represent different CLIMBER-2 runs with varying initial THC strength. Note that for the sake of clarity we restrict our discussion to January (i.e., to the time of intense deep convection in the north), while similar though less pronounced results are obtained for averages over boreal winter or the entire year. The temperature difference $\Delta T \equiv T_{o}-T_{a}$ decreases with time in all simulations

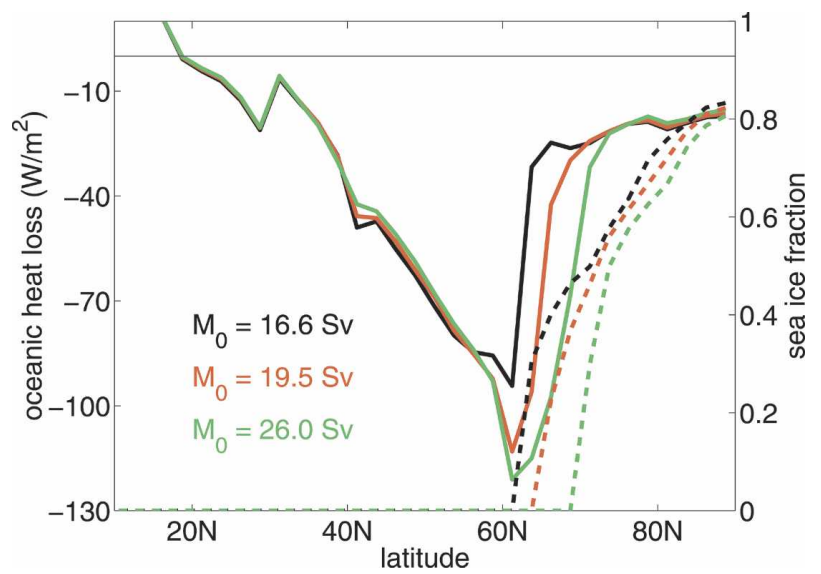

Fig. 4. Heat flux from atmosphere to ocean (full curves) and fraction of sea ice (dashed curves) in the North Atlantic as a function of latitude for different initial strengths of the THC. Negative values of heat flux correspond to a heat loss of the ocean due to a colder SAT. Region of strong oceanic heat loss increases with increasing ice-free area. It is strongest near the sea ice border and small underneath the ice. 


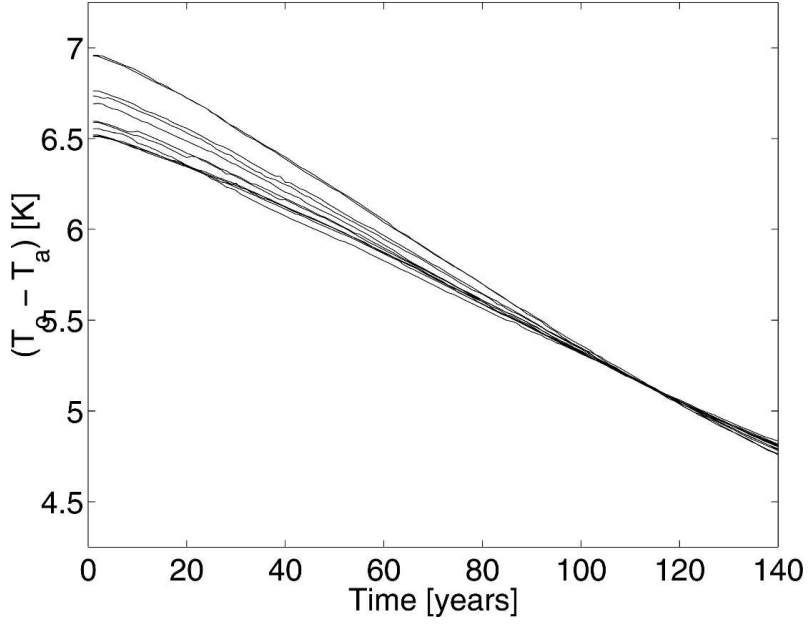

FIG. 5. Time evolution of the temperature difference between ocean and atmosphere $\left(\Delta T \equiv T_{o}-T_{a}\right)$ averaged over the North Atlantic between $20^{\circ}$ and $80^{\circ} \mathrm{N}$ for different initial THC strengths. Initially strong THC leads to stronger $\Delta T$. The difference $\Delta T$ reduces during the $\mathrm{CO}_{2}$ increase mainly due to the atmospheric warming. Because of the ice albedo feedback, $\Delta T$ reduces less for stronger initial THC.

mainly because of atmospheric warming (not shown). This reduces oceanic heat loss [Eq. (1)] and therefore NADW formation. A reduced overturning in turn transports less heat northward and thereby further reduces $\Delta T$. This positive feedback loop (hereafter "temperature effect") is schematized in Fig. 6: an increase in atmospheric $\mathrm{CO}_{2}$ reduces $\Delta T$, which reduces heat loss and THC strength and thereby $\Delta T$.

Changes in near-surface temperature also affect the oceanic heat loss indirectly through the melting of sea ice. This effect is represented as the lower feedback loop in Fig. 6 (hereafter "sea ice effect"). Sea ice retreat due to atmospheric warming will expose a larger area of the ocean to the atmosphere (Fig. 7), which is significantly colder than sea ice in high northern latitudes. The ocean thus loses more heat (Fig. 4), which favors the formation of NADW and tends to counteract the direct temperature effect (i.e., it tends to stabilize the circulation by limiting the reduction in overturning). The time series of ice-free area between $60^{\circ}$ and $80^{\circ} \mathrm{N}$ shows this increase due to rising atmospheric temperatures (Fig. 7). These are the two primary effects of increasing $\mathrm{CO}_{2}$ concentration on the THC: reduction due to warming of the atmosphere and stabilization due to retreating sea ice.

Note that atmospheric temperature does not increase linearly with time in the standard experiment and neither does the ice-free area. The transient relationship between atmospheric warming and sea ice cover, however, is quasi-linear (cf. Fig. 8a). Such model behavior is consistent with simulations of more complex models, which seems to indicate a linear relationship between surface warming and sea ice extent in transient experiments (e.g., Rind et al. 1995; Gregory et al. 2002; Holland and Bitz 2003). We will now investigate the influence of the initial equilibrium on this transient response.

\section{b. Influence of initial THC strength on primary effects}

Next, let us consider the influence of the initial THC strength on these processes (schematized in Fig. 6). An

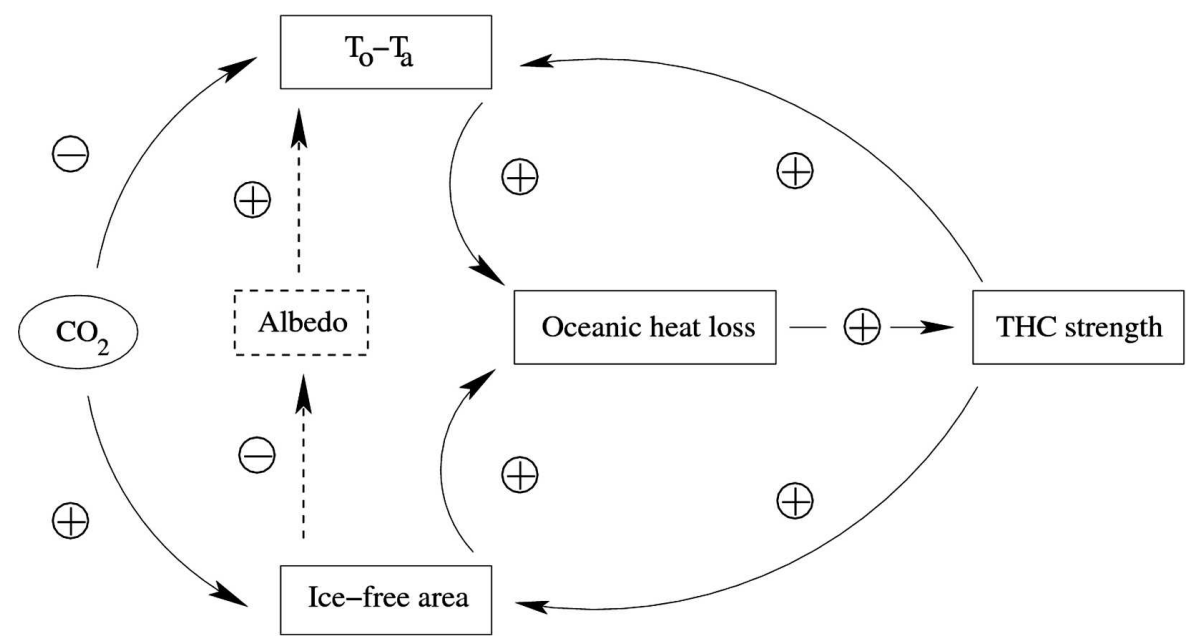

FIG. 6. Causal structure, illustrating the influence of an increase of atmospheric $\mathrm{CO}_{2}$ concentration on the THC. An encircled plus denotes a positive effect of one quantity onto another, a minus a negative one. The physical quantities were computed as boreal wintertime averages in the Atlantic sector between $20^{\circ}$ and $80^{\circ} \mathrm{N}$. 


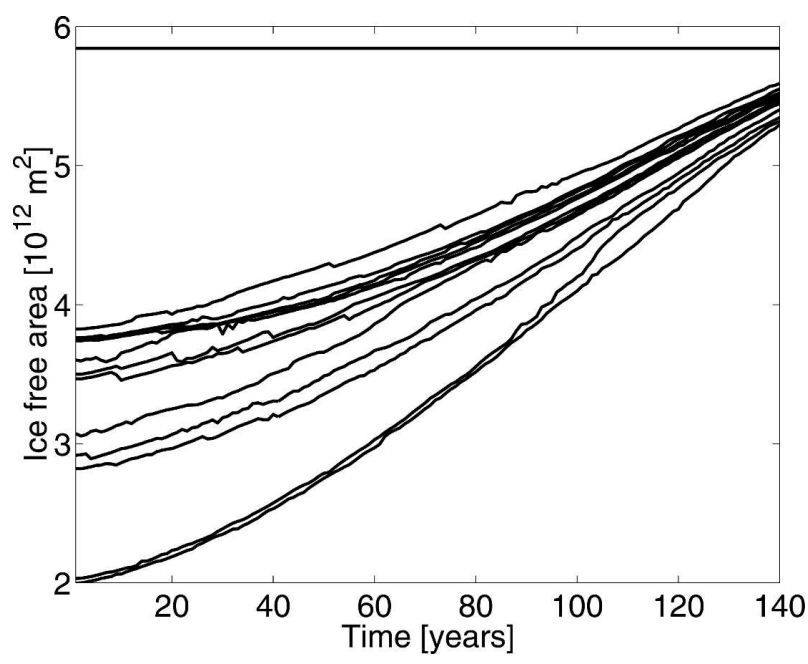

FIG. 7. Time series of the ice-free area $A$ in the North Atlantic between $60^{\circ}$ and $80^{\circ} \mathrm{N}$ for different initial THC strengths. The entire area is given as the upper solid line at $A_{O} \approx 5.8 \times 10^{12} \mathrm{~m}^{2}$.

initially weak THC is associated with an initially large sea ice cover. Figure 8 shows that an initially large sea ice area is more sensitive to atmospheric warming than an initially smaller sea ice cover. This has two main reasons. First, the width of the Nordic Seas decreases with latitude. Consequently, a retreat of the sea ice margin northward yields more ice-free area the farther south the margin initially was. Second, the ice albedo feedback leads to stronger relative warming for an initially larger sea ice cover (cf. Fig. 5), which leads to stronger melting. These two effects lead to the fact that a large initial sea ice cover retreats more strongly than a small one for the same atmospheric warming (Fig. $8 b)$. Thus the influence of the $\mathrm{CO}_{2}$ increase on the sea ice retreat (Fig. 6) depends nonlinearly on the initial THC strength. This modulation of sea ice retreat by the initial THC strength has, in turn, consequences for both temperature effect and sea ice effect, which we will explain in the following.

First, a retreat of sea ice during the warming leads to a reduction of surface albedo, which warms the nearsurface air even further (ice albedo feedback). For a strong initial THC, however, this effect is weak since sea ice extent is already small. Figure $9 \mathrm{a}$, where changes in the temperature difference between ocean and atmosphere are plotted as a function of initial THC strength, illustrates this point: the change in temperature difference is negative, but it decreases in magnitude with increasing initial THC strength. This secondary temperature effect can therefore contribute to the reduction in overturning strength. However, its modulation with $M_{0}$ cannot explain the phenomenon of a
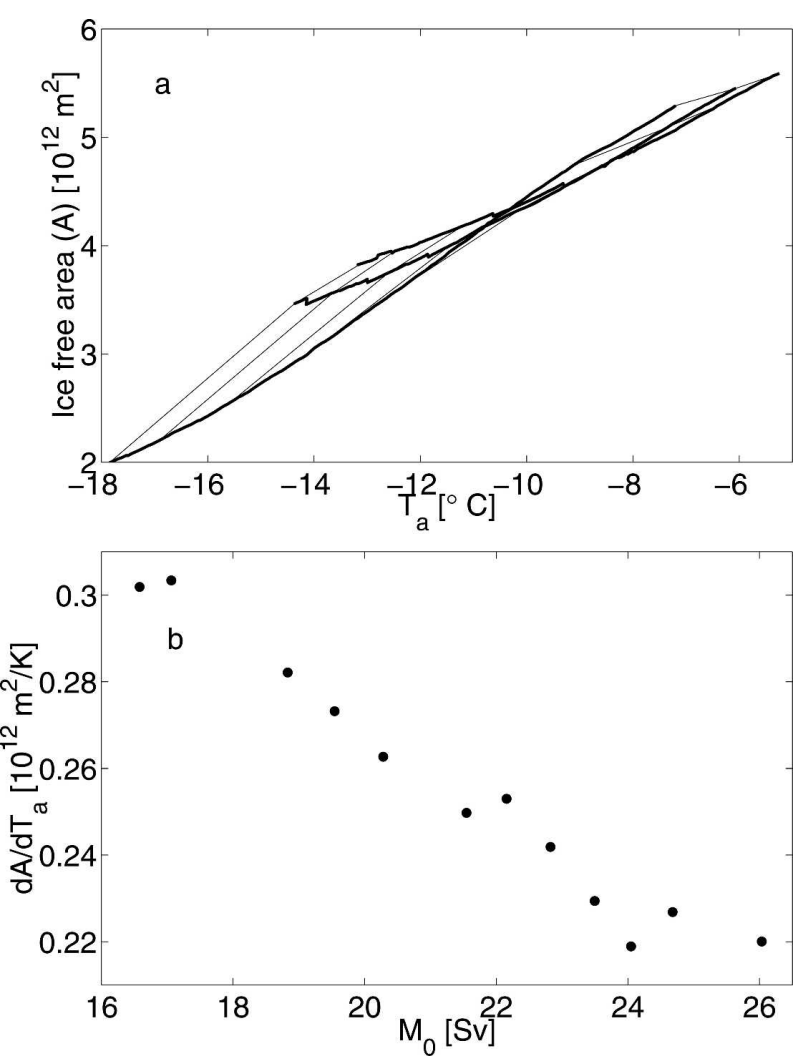

FIG. 8. (a) Ice-free area as a function of SAT in the North Atlantic for three simulations with different initial strengths of the THC. The SAT was taken in January between $20^{\circ}$ and $80^{\circ} \mathrm{N}$. The thick lines give the evolution of the ice-free area with time as a function of SAT. The thin lines are meant to guide the reader's eye and connect different corresponding times in the different runs with an interval of $10 \mathrm{yr}$. (b) Change in ice-free area per kelvin during the global warming experiments as a function of the initial strength of the THC, given as the average slope of the lines in (a). The sensitivity of the ice-free area to changes in SAT decreases with increasing initial THC.

strong weakening of an initially strong THC seen in Fig. 2.

Second, the effect of initial THC strength on the sea ice effect can be seen in Fig. 9b: like the temperature effect, it is also reduced for strong initial THC. In this case, however, it has the inverse influence on the THC weakening, because the sea ice effect tends to strengthen the THC (Fig. 6). Thus the modulation of the THC weakening through the sea ice effect has the correct qualitative behavior to explain the sign of the slope in Fig. 2.

A consequence of the above reasoning is that the THC should weaken less in models with strong sea ice retreat $\triangle A$ during atmospheric warming. This is indeed the case for the presented CLIMBER-2 simulations (Fig. 10a). Figure 10b shows the change in ice-free area as a function of THC weakening for the models par- 

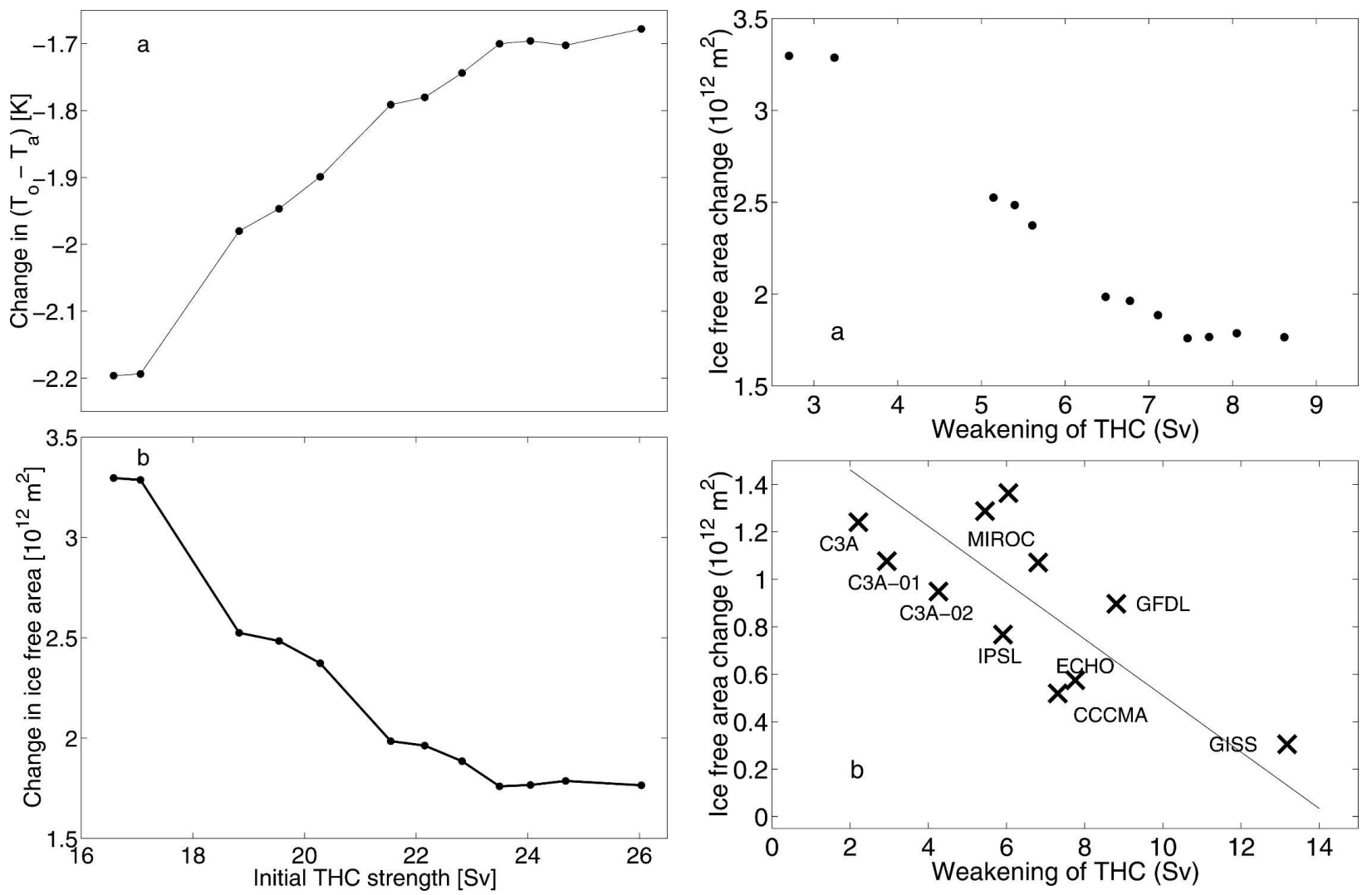

FIG. 9. Change in (a) $\Delta T$ and (b) $A$ between the end and beginning of the scenario as a function of initial THC strength (both taken for January between $20^{\circ}$ and $80^{\circ} \mathrm{N}$ ). Note that the change in $\Delta T<0$ is negative (see also Fig. 5); that is, atmospheric and oceanic temperature are closer after the $\mathrm{CO}_{2}$ increase than before, but this effect is less pronounced for stronger initial THC. The change in $A$ is positive mainly due to the melting of sea ice in the course of the scenario. This effect is also less pronounced for stronger initial THC (cf. Fig. 7).

ticipating in the IPCC AR4 intercomparison and for the CLIMBER-3 $\alpha$ simulations. (Unfortunately, sea ice data are not available for the CMIP.) These models, which all include an OGCM, show the same qualitative behavior as CLIMBER-2. The correlation coefficient of a linear regression is $r=-0.72$. The ice-free area was computed between $60^{\circ}-80^{\circ} \mathrm{N}$ and $45^{\circ} \mathrm{W}-35^{\circ} \mathrm{E}$. In the zonally averaged architecture of the CLIMBER-2 ocean model, longitude cannot be constrained in the computation of $\Delta A$. This prevents quantitative comparison of the ice-free areas with models containing an OGCM. Associated limitations for the generalization to more complex models will be discussed in section 6 .

\section{c. Regional changes in oceanic heat loss}

The two effects of rising atmospheric temperatures and retreating sea ice are combined in the oceanic heat

FIG. 10. Change in ice-free area as a function of THC weakening after $140 \mathrm{yr}$ of $\mathrm{CO}_{2}$ increase for the (a) CLIMBER-2 simulations and for (b) models participating in the IPCC AR4 intercomparison and the CLIMBER-3 $\alpha$ model. Models including an (b) OGCM show the same qualitative behavior as (a) CLIMBER-2 [i.e., a stronger weakening of the THC is associated with a smaller change in ice-free area (between $60^{\circ}-80^{\circ} \mathrm{N}$ and $45^{\circ} \mathrm{W}-30^{\circ} \mathrm{E}$ )]. Linear regression results in a correlation coefficient of $r=-0.75$. Strongest deviations occur for the three Model for Interdisciplinary Research on Climate (MIROC) experiments. The linear regression excluding MIROC yields the plotted line and $r=-0.91$.

loss evolution (Fig. 11a: difference between values after $140 \mathrm{yr}$ and initial values; Fig. 11b: the same for the final equilibrium). The change in January oceanic heat loss in the area between $20^{\circ}$ and $80^{\circ} \mathrm{N}$ (curve with diamonds) is negative, explaining the weakening of the THC in the course of the $\mathrm{CO}_{2}$ increase. It is furthermore stronger in magnitude for initially stronger overturning, which explains the stronger weakening of the THC in this case (Fig. 2). By separating permanently ice-free areas $\left(20^{\circ}-60^{\circ} \mathrm{N}\right.$; curve with stars) from regions affected by changes in sea ice cover $\left(60^{\circ}-80^{\circ} \mathrm{N}\right.$; curve with solid circles), we can identify the effects dominating the sign and the slope of the curve with diamonds in Fig. 11. The change in heat loss is negative for the more southern region, which determines the sign for the en- 

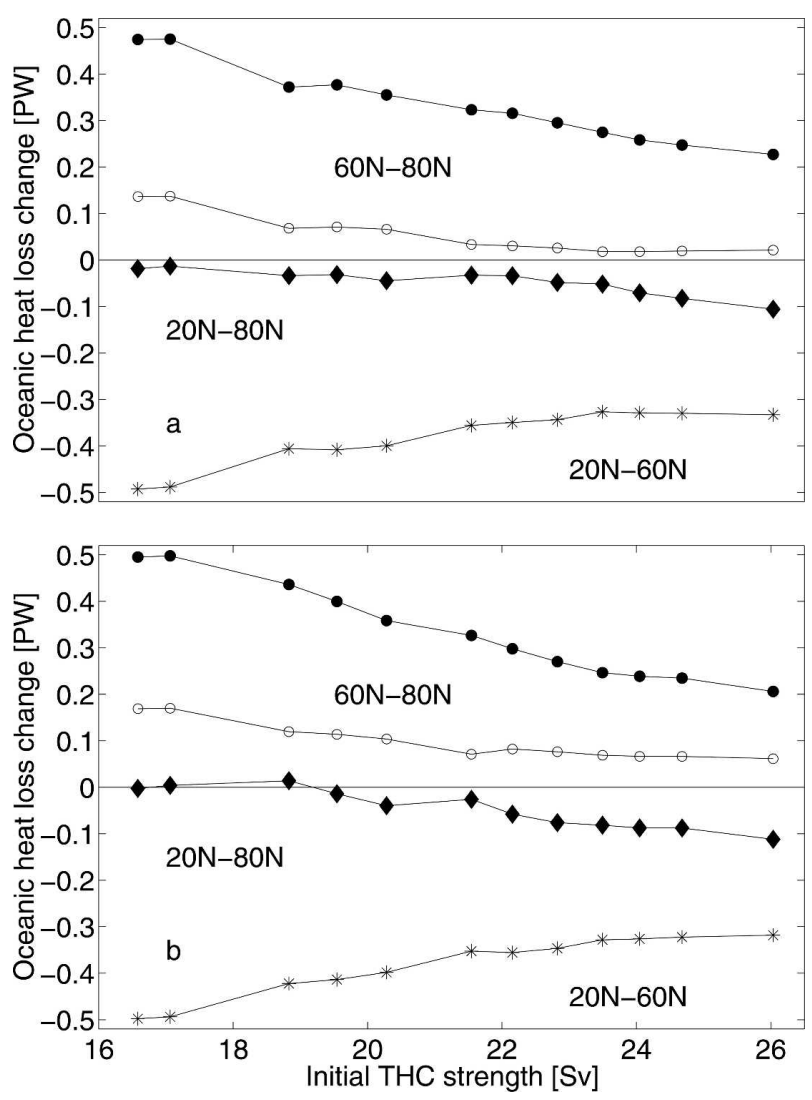

FIG. 11. Change in oceanic heat loss in January as a function of initial THC strength. In areas unaffected by changes in sea ice $\left(20^{\circ}-60^{\circ} \mathrm{N}\right.$; curve with stars), the change in heat loss is negative and decreases in magnitude. In northern regions $\left(60^{\circ}-80^{\circ} \mathrm{N}\right.$; dashed curve with open circles), the change in heat loss is positive (because of an increase in ice-free area) and decreases with initial THC strength. The entire change in heat loss $\left(20^{\circ}-80^{\circ} \mathrm{N}\right.$; curve with diamonds) explains both sign and slope of the dependence of THC weakening on $M_{0}$ found in Fig. 2. The change in latent heat loss in the northern region (curve with open circles) shows the same behavior as the total net heat loss (curve with filled circles). Differences between (a) 140-yr and initial values and (b) final and initial equilibria.

tire North Atlantic and is consistent with the decrease in $T_{o}-T_{a}$ (Fig. 5). (Note that the responsible ice albedo effect is strongest near the sea ice margin, but influences the permanently ice-free region between $20^{\circ}$ and $60^{\circ} \mathrm{N}$.) However, due to the dependence of $T_{o}-T_{a}$ on $M_{0}$, heat loss changes are weaker for initially stronger THC in this area, so that as detailed before, the slope of the curve with open circles cannot explain that of Fig. 2. In contrast, heat loss for the partially icecovered region $\left(60^{\circ}-80^{\circ} \mathrm{N}\right)$ shows an increase in the course of the scenario due to the increase of ice-free area during the warming (Fig. 7). This effect is also weaker for initially stronger THC but has the reversed sign compared to the temperature effect and thus tends to stabilize NADW formation and THC strength. In summary, the change in heat loss for the entire North Atlantic $\left(20^{\circ}-80^{\circ} \mathrm{N}\right.$; curve with diamonds) has the negative sign of the temperature effect, while its slope is given by the sea ice effect.

\section{Qualitative discussion of the sign of the slope}

Purely in the line of reasoning of section 4 (i.e., ignoring the possible role of freshwater fluxes), we can further discuss the robustness of the described mechanism. To better understand the sign of the slopes in Fig. 2, let us reconsider the schematic of Fig. 6. In section 4 we argue that the dependence on the initial strength of the THC enters through the initial ice-free area, which influences the rate of sea ice retreat under global warming. More initial sea ice cover retreats more strongly. The sign of the slope is then determined by the competition between the direct effect of the retreating sea ice on the heat loss and the indirect effect via the albedo change and the resulting change in temperature difference between ocean and atmosphere. This can be expressed mathematically in terms of derivatives, following the two respective paths in Fig. 6. In this view, the sign of the slope is determined by the dependence of the heat loss $H$ on the ice-free area $A$, that is, by the sign of

$$
\frac{d H}{d A}=\left(\frac{\partial H}{\partial A}\right)_{\Delta T}+\left(\frac{\partial H}{\partial \Delta T}\right)_{A} \times \frac{\partial \Delta T}{\partial \bar{\alpha}} \times \frac{\partial \bar{\alpha}}{\partial A},
$$

with $\Delta T=T_{o}-T_{a}$. The first term on the right-hand side describes the direct effect of reduced sea ice area on the heat loss; the second term gives the indirect effect through the change of surface albedo. Here $\bar{\alpha}=$ $\left[A \times \alpha_{O}+\left(A_{O}-A\right) \times \alpha_{I}\right] / A_{O}$ is the surface albedo averaged over the North Atlantic region of oceanic heat loss with $a_{I}$ and $\alpha_{O}$ being the albedo of sea ice and ocean, respectively. $A_{O}$ is the entire area of ocean heat loss in the North Atlantic irrespective of the sea ice cover. In the framework of section 4, that is the Atlantic area between $20^{\circ}$ and $80^{\circ} \mathrm{N}$. For

$$
\left(\frac{\partial H}{\partial A}\right)_{\Delta T}>\left(\frac{\partial H}{\partial \Delta T}\right)_{A} \times \frac{\partial \Delta T}{\partial \bar{\alpha}} \times \frac{\alpha_{I}-\alpha_{O}}{A_{O}},
$$

the full derivative in Eq. (2) is positive and so is the slope in Fig. 2. If we assume that Eq. (1) approximates the oceanic heat loss, we can simplify this relation to

$$
\overline{\Delta T}>\frac{A}{A_{O}} \times \frac{\partial \Delta T}{\partial \bar{\alpha}} \times\left(\alpha_{I}-\alpha_{O}\right) .
$$

We write here $\overline{\Delta T}$ in order to emphasize that the temperature difference between ocean and atmosphere is 
averaged over the North Atlantic area of oceanic heat loss. Equation (4) is written for a specific time. To make a statement about the slope of the curves in Fig. 2, the relation has to hold throughout the simulation. Since $A$ cannot be larger than $A_{O}$ and $\left(\alpha_{I}-\alpha_{O}\right)$ cannot be larger than 1 , we can say that relation (4) holds when

$$
\overline{\Delta T}>\frac{\partial \Delta T}{\partial \bar{\alpha}} .
$$

This means that a strong initial THC weakens more under global warming as long as the temperature difference between ocean and atmosphere averaged over the North Atlantic area of oceanic heat loss is larger than the increase of this temperature difference due to a change of surface albedo from that of sea ice to that of the ocean. In this case the direct effect of ice melting dominates over the indirect albedo effect.

Note that the quantity $\partial \Delta T / \partial \bar{\alpha}$ is highly model dependent and involves a number of complicated physical processes. The slope in Fig. 2 indicates that in CLIMBER-2 it is bounded by $4.8 \mathrm{~K}$, the lower bound of $\overline{\Delta T}$ in the simulations (Fig. 5). Indeed, experiments with CLIMBER-2 have shown that surface air temperature increases by only about $3 \mathrm{~K}$ when the albedo of sea ice is set artificially to that of the ocean (A. Ganopolski 2005 , personal communication), consistent with the observed behavior.

\section{Discussion and conclusions}

Based on a number of model simulations with the EMIC CLIMBER-2 and an idealized $\mathrm{CO}_{2}$-increase scenario ( $1 \%$ yearly increase up to $1120 \mathrm{ppm}$ ), we propose a sea ice-related process that tends to stabilize the THC under atmospheric warming. This mechanism is weaker for initially stronger THC and is identified to dominate the dependence of THC changes on the initial THC strength in CLIMBER-2.

The CMIP intercomparison project showed that a stronger initial THC weakens more under global warming. Experiments with CLIMBER-2 and CLIMBER-3 $\alpha$ exhibit a linear relation between THC weakening and initial strength (section 3). The participating models of the CMIP, EMIP, and IPCC AR4 intercomparison projects scatter around this line formed by the CLIMBER simulations. For the CMIP experiments it was shown that the THC weakening is dominated by changes in heat flux as opposed to freshwater fluxes (Gregory et al. 2005). This effect is specifically pronounced in CLIMBER-2. We therefore use additional experiments with CLIMBER-2 to examine heat fluxrelated processes that are relevant under warming and investigate their dependence on the initial THC strength.

The THC weakening is primarily due to a warming of atmospheric temperatures (Fig. 11) and the associated reduction in oceanic heat loss in the North Atlantic. For CLIMBER-2, this effect is shown to be weaker for initially strong THC because of the ice albedo effect (Fig. 9). It thus cannot explain the described dependence that an initially stronger THC weakens more. A second important effect that occurs under warming is the melting of sea ice in the North Atlantic and Arctic Ocean. This leads to an increase in oceanic heat loss due to a larger ice-free area (i.e., main area, over which the ocean loses heat to the atmosphere). This effect tends to increase deep-water formation in the Nordic Seas and strengthen the THC. It is weaker for an initially stronger THC and was identified to dominate the overall dependence of the THC weakening on its initial strength in CLIMBER-2. We argue that this is the case as long as the temperature difference between ocean and atmosphere in the North Atlantic $\Delta T$ is larger than the potential change in $\Delta T$ due to a change in surface albedo from its oceanic value to its sea ice value (section 5).

As a consequence of the warming, the oceanic heat loss exhibits a decrease in North Atlantic regions that are not affected by sea ice cover $\left(20^{\circ}-60^{\circ} \mathrm{N}\right)$. On the other hand, it increases in regions of strong sea ice retreat $\left(60^{\circ}-80^{\circ} \mathrm{N}\right)$. The latter is more pronounced for initially larger sea ice cover, which is associated with a weak initial THC.

An important question is whether the mechanism found in CLIMBER-2 is relevant to other models. There are some indications. Saenko et al. (2004) already argued that northern sea ice cover is crucial for the behavior of the THC under warming. Even though there is no direct relation between THC weakening and initial strength in their simulations because of their specific experimental setup, their experiments are consistent with the mechanism presented here. In particular, they find a strengthening of the THC under warming in an experiment with unrealistically large initial sea ice cover. They show that in this case, the net buoyancy flux into the ocean further decreases in the course of the experiment, which was caused mainly by thermal fluxes. This would be consistent with a strong increase in ice-free area although this is not shown in their study.

Furthermore, the scattering of the CMIP, EMIP, and IPCC AR4 simulations around the CLIMBER experiments indicates that there is a general tendency for a stronger initial THC to weaken more under warming. The presented mechanism shows this qualitative behavior, while a mere change in atmospheric temperature 
without additional heat loss in melting regions would lead to the inverse dependence (ice albedo feedback). Note further that some of the scatter in Fig. 2 is likely due to internal variability in some of the AOGCMs of the CMIP and IPCC AR4 intercomparisons. Third, the mechanism discussed in this study is based on heat flux changes under warming, which were shown to dominate THC changes in all CMIP simulations. We concede that this dominance was particularly pronounced in CLIMBER-2 and therefore the proposed mechanism might be stronger in CLIMBER-2 compared to other models. But it is consistent with the findings of the CMIP, EMIP, and IPCC AR4 simulations presented. Unfortunately, no sea ice data were available from the CMIP intercomparison. Models from the IPCC AR4, however, show that a stronger weakening of the THC is indeed associated with less sea ice melt (Fig. 10). This dependence is also found in CLIMBER-2 and is central to the mechanism. This is a fourth indication that the mechanism might be relevant to other models.

The above, however, is not sufficient to claim that the sea ice process is dominant or even at play in other models. There are a number of strong limitations to a generalization of the results. First of all there is the possible influence of changes in freshwater fluxes. Though Gregory et al. (2005) showed that changes in freshwater fluxes are not dominant in the CMIP models, they are not negligible in all models and might be of the same order as the heat flux changes in some experiments. This point is specifically important when including melting of the Greenland ice sheet.

Other possible limitations result directly from the zonally averaged oceanic component of CLIMBER-2. As a consequence, CLIMBER-2 does not distinguish deep-water formation in the Labrador Sea and Nordic Seas. Possible switches between these convection sites that might occur under warming are therefore not captured. Furthermore, changes in ice-free areas are quantitatively different in CLIMBER-2 compared to OGCMs (Fig. 10), which might lead to an overestimation of the described mechanism. CLIMBER-3 $\alpha$, however, does not suffer the shortcomings of a zonally averaged ocean and shows the same qualitative behavior of the overturning weakening under warming. The statistical-dynamical atmosphere component of both CLIMBER models might induce some inherent limitations to atmospheric transport processes (including the lack of atmospheric variability). This might have implications for atmospheric heat and freshwater transport, oceanic mixing processes, and the relative importance of changes in heat and freshwater forcing to the ocean under warming. The representation of sea ice physics could play a role for the mechanism in different models and in the real ocean.

Independent of the quantitative applicability of the CLIMBER-2 results for other models, the physical arguments presented have the potential to qualitatively explain the observed phenomenon and play a role for the THC stability under warming. A better and more quantitative understanding of the mechanism could lead to improved projections of future THC evolution, since changes in sea ice cover and surface fluxes are better constraints than the deep volume fluxes associated with the THC.

Acknowledgments. We thank V. Petoukhov for providing the EMIP data, C. Covey for assistance with the IPCC AR4 database, and an anonymous reviewer for valuable comments. A. Levermann and J. Mignot were funded by the G. Comer foundation. S. Nawrath was funded through the James S. McDonnell Foundation Centennial Fellowship.

\section{REFERENCES}

Alley, R. B., P. U. Clark, P. Huybrechts, and I. Joughin, 2005: Ice-sheet and sea-level changes. Science, 310, 456-460.

Bond, G., and Coauthors, 1992: Evidence for massive discharge of icebergs into the North Atlantic ocean during the last glacial period. Nature, 360, 245-249.

Clark, P. U., N. G. Pisias, T. F. Stocker, and A. J. Weaver, 2002: The role of the thermohaline circulation in abrupt climate change. Nature, 415, 863-869.

Crowley, T. J., 1992: North Atlantic Deep Water cools the southern hemisphere. Paleoceanography, 7, 489-497.

Fichefet, T., and M. A. M. Maqueda, 1997: Sensitivity of a global sea ice model to the treatment of ice thermodynamics and dynamics. J. Geophys. Res., 102, 12 609-12 646.

- C. Poncin, H. Goosse, P. Huybrechts, I. Janssens, and H. Le Treut, 2003: Implications of changes in freshwater flux from the Greenland ice sheet for the climate of the 21st century. Geophys. Res. Lett., 30, 1911, doi:10.1029/2003GL017826.

Ganachaud, A., and C. Wunsch, 2000: Improved estimates of global ocean circulation, heat transport and mixing from hydrographic data. Nature, 408, 453-457.

_ ports during the World Ocean Circulation Experiment. $J$. Climate, 16, 696-705.

Ganopolski, A., and S. Rahmstorf, 2001: Rapid changes of glacial climate simulated in a coupled climate model. Nature, 409, 153-158.

Gregory, J. M., P. A. Stott, D. J. Cresswell, N. A. Rayner, C. Gordon, and D. M. H. Sexton, 2002: Recent and future changes in Arctic sea ice simulated by the HadCM3 AOGCM. Geophys. Res. Lett., 29, 2175, doi:10.1029/2001GL014575.

_ - and Coauthors, 2005: A model intercomparison of changes in the Atlantic thermohaline circulation in response to increasing atmospheric $\mathrm{CO}_{2}$ concentration. Geophys. Res. Lett., 32, L12703, doi:10.1029/2005GL023209.

Hall, M. M., and H. L. Bryden, 1982: Direct estimates and mechanisms of ocean heat transport. Deep-Sea Res., 29, 339-359. 
Hansen, J. E., 2005: A slippery slope: How much global warming constitutes "dangerous anthropogenic interference"? Climatic Change, 68, 269-279.

Herweijer, C., R. Seager, M. Winton, and A. Clement, 2005: Why ocean heat transport warms the global mean climate. Tellus, 57, 662-675.

Holland, M. M., and C. M. Bitz, 2003: Polar amplification of climate change in coupled models. Climate Dyn., 21, 221-232.

Houghton, J. T., Y. Ding, D. J. Griggs, M. Noguer, P. J. van der Linden, X. Dai, K. Maskell, and C. A. Johnson, Eds., 2001: Climate Change 2001: The Scientific Basis. Cambridge University Press, $881 \mathrm{pp}$.

Kubatzki, C., M. Montoya, S. Rahmstorf, A. Ganopolski, and M. Claussen, 2000: Comparison of the last interglacial climate simulated by a coupled global model of intermediate complexity and an AOGCM. Climate Dyn., 16, 799-814.

Levermann, A., A. Griesel, M. Hofmann, M. Montoya, and S. Rahmstorf, 2005: Dynamic sea level changes following changes in the thermohaline circulation. Climate Dyn., 24, 347-354.

Manabe, S., and R. J. Stouffer, 1988: Two stable equilibria of a coupled ocean-atmosphere model. J. Climate, 1, 841-866.

— , and - 1997: Coupled ocean-atmosphere model response to freshwater input: Comparision to Younger Dryas event. Paleoceanography, 12, 321-336.

McManus, J. F., R. Francois, J. M. Gherardi, L. D. Keigwin, and S. Brown-Leger, 2004: Collapse and rapid resumption of Atlantic meridional circulation linked to deglacial climate changes. Nature, 428, 834-837.

Mignot, J., A. Levermann, and A. Griesel, 2006: A decomposition of the Atlantic meridional overturning circulation into physical components using its sensitivity to vertical diffusivity. $J$. Phys. Oceanogr., 36, 636-650.

Montoya, M., A. Griesel, A. Levermann, J. Mignot, M. Hofmann, A. Ganopolski, and S. Rahmstorf, 2005: The Earth System Model of Intermediate Complexity CLIMBER-3 $\alpha$. Part I: Description and performance for present day conditions. Climate Dyn., 25, 237-263.

Oppenheimer, M., and R. B. Alley, 2005: Ice sheets, global warming and Article 2 of the UNFCCC. Climatic Change, 68, 257267.

Petoukhov, V., A. Ganopolski, V. Brovkin, M. Claussen, A. Eliseev, C. Kubatzki, and S. Rahmstorf, 2000: CLIMBER-2: A climate system model of intermediate complexity. Part I: Model description and performance for present climate. Climate Dyn., 16, 1-17.

-, and Coauthors, 2005: EMIC Intercomparison Project (EMIP- $\mathrm{CO}_{2}$ ): Comparative analysis of EMIC simulations of climate and of equilibrium and transient responses to atmospheric $\mathrm{CO}_{2}$ doubling. Climate Dyn., 25, 363-385.
Rahmstorf, S., 2002: Ocean circulation and climate during the past 120000 years. Nature, 419, 207-214

—_, and A. Ganopolski, 1999: Long-term global warming scenarios computed with an efficient coupled climate model. Climatic Change, 43, 353-367.

Rind, D., R. Healy, C. Parkinson, and D. Martinson, 1995: The role of sea ice in $2 \times \mathrm{CO}_{2}$ climate model sensitivity. Part I: The total influence of sea ice thickness and extent. J. Climate, 8, 449-463.

Saenko, O. A., M. Eby, and A. J. Weaver, 2004: The effect of sea-ice extent in the North Atlantic on the stability of the thermohaline circulation in global warming experiments. Climate Dyn., 22, 689-699.

Schmittner, A., 2005: Decline of the marine ecosystem caused by a reduction in the Atlantic overturning circulation. Nature, 434, 628-633.

Semtner, A., 1976: A model for the thermodynamic growth of sea ice in numerical investigations of climate. J. Phys. Oceanogr., 6, 379-389.

Stocker, T. F., 1998: The seesaw effect. Science, 282, 61-62.

Stouffer, R. J., and Coauthors, 2006: Investigating the causes of the response of the thermohaline circulation to past and future climate changes. J. Climate, 19, 1365-1387.

Talley, L. D., J. L. Reid, and P. E. Robbins, 2003: Data-based meridional overturning streamfunctions for the global ocean. J. Climate, 16, 3213-3226.

Timmermann, A., S. I. An, U. Krebs, and H. Goosse, 2005: ENSO suppression due to weakening of the North Atlantic thermohaline circulation. J. Climate, 18, 2842-2859.

Vellinga, M., and R. A. Wood, 2002: Global climatic impacts of a collapse of the Atlantic thermohaline circulation. Climatic Change, 54, 251-267.

von Deimling, S. T., H. Held, A. Ganopolski, and S. Rahmstorf, 2006: Climate sensitivity estimated from ensemble simulations of glacial climate. Climate Dyn., 27, 149-163.

Winguth, A., U. Mikolajewicz, M. Gröger, E. Maier-Reimer, G. Schurgers, and M. Vizcaíno, 2005: Centennial-scale interactions between the carbon cycle and anthropogenic climate change using a dynamic Earth system model. Geophys. Res. Lett., 32, L23714, doi:10.1029/2005GL023681.

Winton, M., 2003: On the climatic impact of ocean circulation. $J$. Climate, 16, 2875-2889.

Wood, R. A., A. B. Keen, J. F. B. Mitchell, and J. M. Gregory, 1999: Changing spatial structure of the thermohaline circulation in response to atmospheric $\mathrm{CO}_{2}$ forcing in a climate model. Nature, 399, 572-575.

Zickfeld, K., A. Levermann, H. M. Granger, S. Rahmstorf, T. Kuhlbrodt, and D. W. Keith, 2007: Current state and future fate of the ocean circulation as viewed by experts. Climatic Change, 82, 235-265. 